\title{
Direction-specific Release from Capsules with Homogeneous or Janus Shells Using an Ultrasound Approach
}

Tomasz Kubiak, ${ }^{\ddagger}$ Joanna Banaszak, ${ }^{\S}$ Arkadiusz Józefczak, ${ }^{\S}$ and Zbigniew Rozynek ${ }^{\dagger{ }^{*}}$

$\S$ Institute of Acoustics, Faculty of Physics, Adam Mickiewicz University, Uniwersytetu Poznańskiego 2, 61-614 Poznań, Poland

$\$$ Hipolit Cegielski State University of Applied Sciences, Stefana Wyszyńskiego 38, 62-200 Gniezno, Poland

$\dagger$ Harvard John A. Paulson School of Engineering and Applied Sciences, Harvard University, Cambridge, MA 02138, USA

${ }^{*}$ Corresponding author: zbiroz@amu.edu.pl

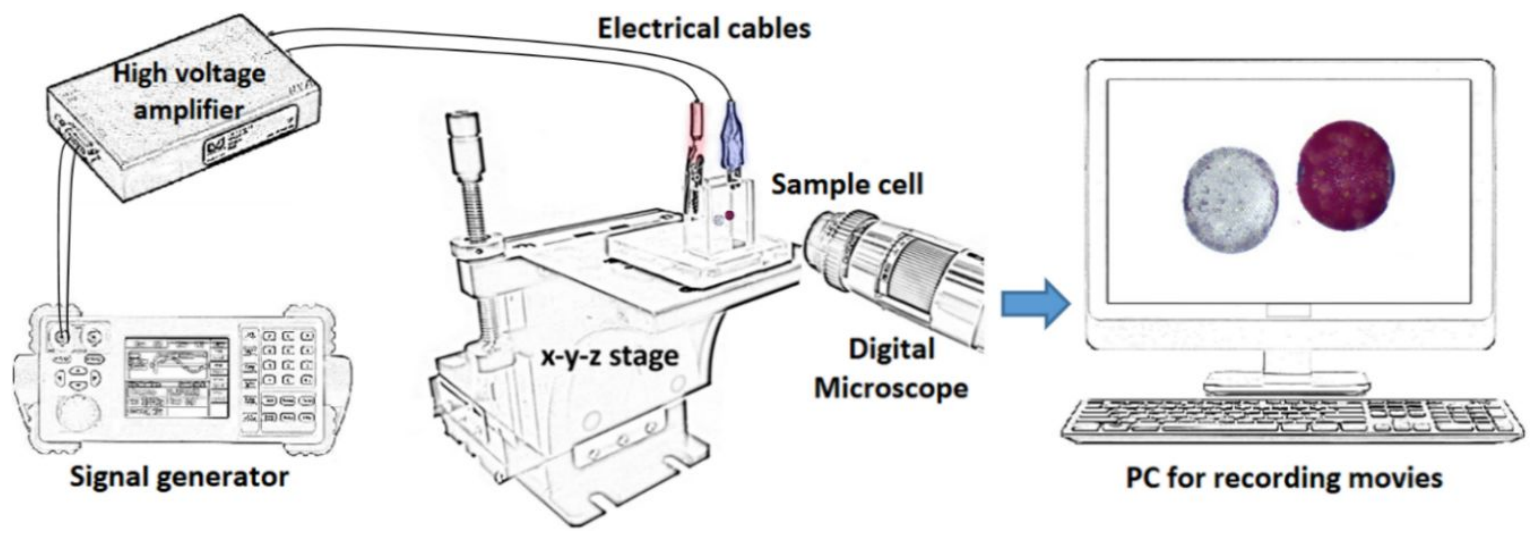

Figure S1. A schematic illustration of the experimental set-up, consisting of a sample cell placed on a mechanical XYZ translation stage, a digital microscope for viewing in the direction perpendicular to the electric field direction, a signal generator and a voltage amplifier for generating high-voltage signals, and a computer for recording videos.
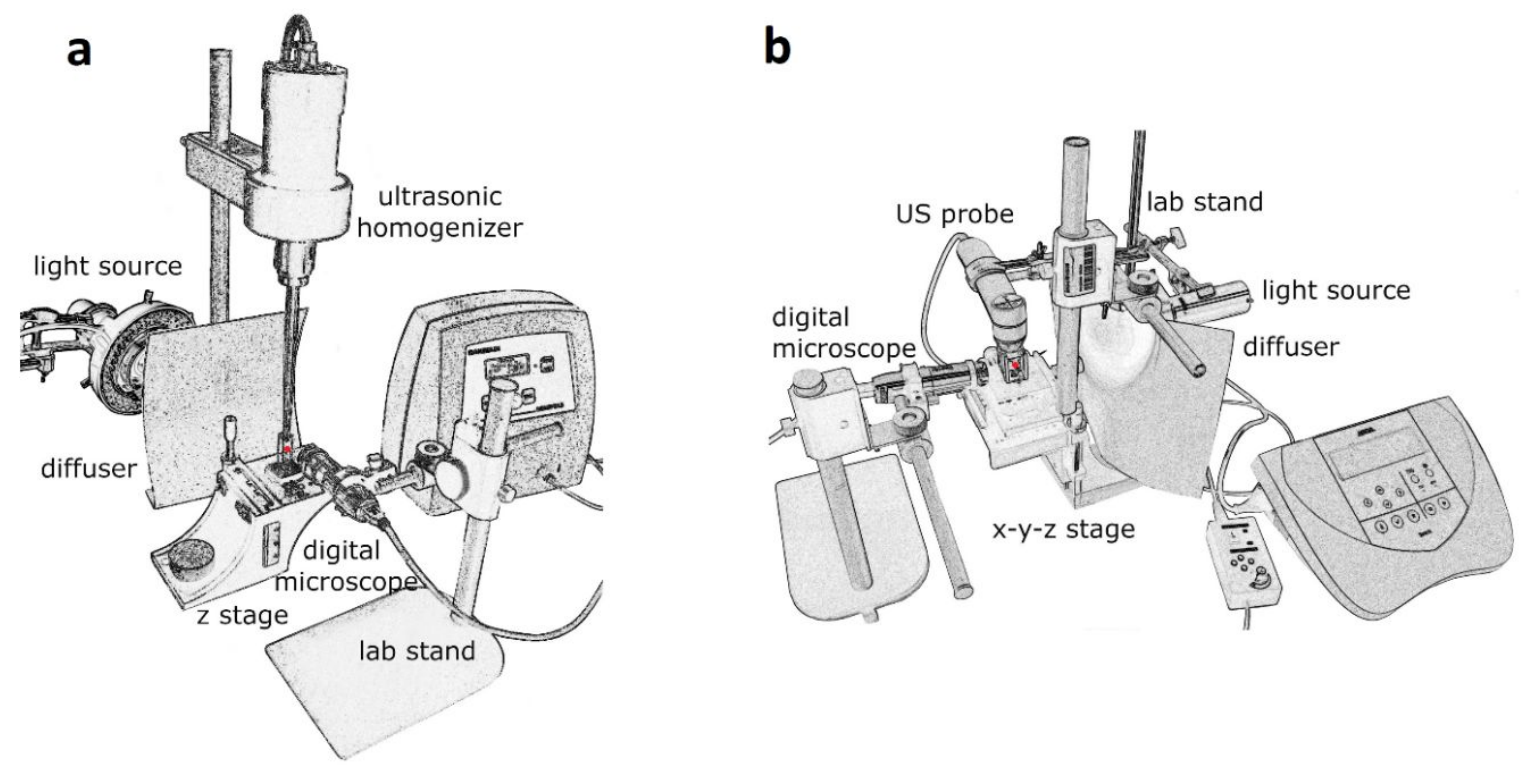

Figure S2. We used two set-ups for studying ultrasound-induced rupturing of capsules. (a) Low frequency $(20 \mathrm{kHz})$ ultrasound impulses were generated by Sonopuls ultrasonic homogenizer HD 3100 from Bandelin electronic GmbH \& Co. (b) High frequency (1 MHz) ultrasound waves were generated by Sonaris S from Astar. 

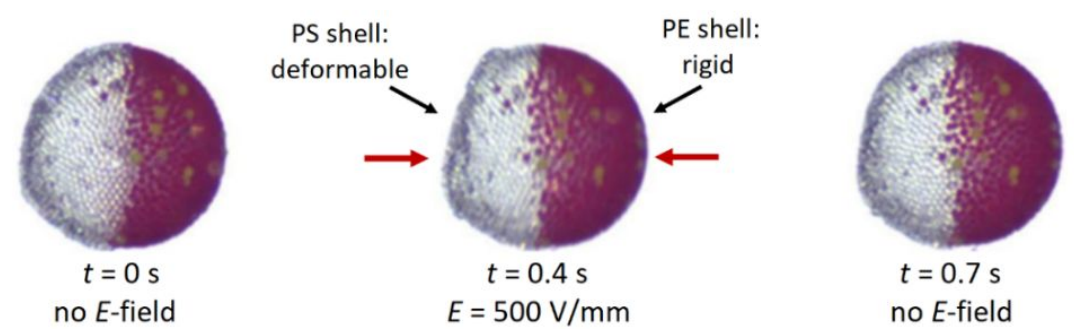

Figure S3. We used an electric field, as a non-contact method, to verify whether particle shells of thermally treated droplets were mechanically stable. We applied an electric field to induce electric stress on the capsules. (left) PS-PE Pickering droplet after thermal treatment in microwave oven for $\sim 15$ seconds. (middle) Electric stress deformed the left side of the droplet with the shell composed of non-interlocked PS particles, whereas the shell made of interlocked PE particles stayed undeformed owing to its rigidity. (right) After turning off the electric field $(t=0.5 \mathrm{~s})$, the deformed PS shell relaxed and the particle-covered droplet became spherical again. See also the corresponding Movie S3.

We used an electric field, as a non-contact method, to verify whether particle shells of the thermally treated capsules were mechanically stable. We applied an electric field to induce electric stress on the capsules. When a silicone oil droplet or a droplet covered with weakly conductive particles is suspended in castor oil and subjected to a uniform DC electric field, free charges (ionic impurities in oils) accumulate at the droplet's interface. The electric field exerts force on these charges, resulting in electric stress. For a non-interlocked particle shell the electric stress deforms the drop, whereas shells with interlocked particles stays undeformed owing to the rigidity of such particle shell. In Figure S3 and Movie S3 we demonstrate examples of the performed tests of mechanical properties of shells.

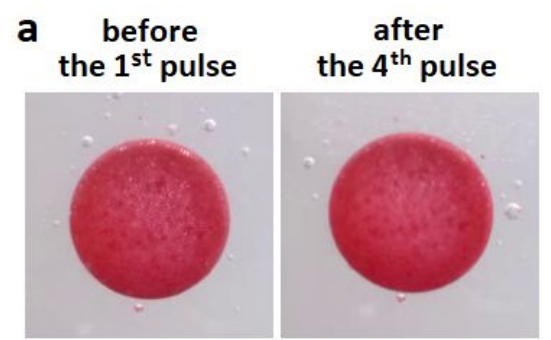

$\underline{20 \mathrm{~W} / \mathrm{cm}^{2}}$

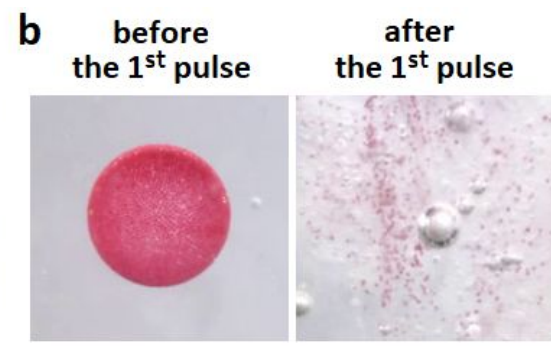

$100 \mathrm{~W} / \mathrm{cm}^{2}$

Figure S4. The influence of $20-\mathrm{kHz}$ ultrasound with the intensity of (a) $\sim 20 \mathrm{~W} / \mathrm{cm}^{2}$ and (b) $\sim 100 \mathrm{~W} / \mathrm{cm}^{2}$ on a Pickering droplet in castor oil. (a)The Pickering droplet subjected to low intensity ultrasound deformed and relaxed back to the spherical shape without particle detachment from the interface. (b) Application of ultrasound with the highest available intensity resulted in immediate and complete fragmentation of the droplet by the first pulse.

Table S1. Particle concentration in silicone oil and droplet sizes.

\begin{tabular}{cccc}
\hline Figure & Particles & $\begin{array}{c}\text { Concentration } \\
{[\mathrm{wt} \%]}\end{array}$ & $\begin{array}{c}\text { Droplet size } \\
{[\mathrm{mm}]}\end{array}$ \\
\hline $2 \mathrm{a}$ & PE50(red) & $\sim 9$ & 2.0 \\
\hline $2 \mathrm{~b}$ & PE50(red) / PS40 & $\sim 8 / \sim 8$ & 1.6 \\
\hline $3 \mathrm{a}$ & PS40 & $\sim 8$ & 1.9 \\
\hline $3 \mathrm{~b}$ & PS40 & $\sim 8$ & 1.9 \\
\hline $3 \mathrm{c}$ & PS40 & $\sim 9$ & 1.8 \\
\hline $3 \mathrm{~d}$ & PE50(red) / PS40 & $\sim 6 / \sim 5$ & 2.7 \\
\hline $4 \mathrm{a}$ & PS40 & $\sim 9$ & 1.8 \\
\hline $4 \mathrm{~b}$ & PS40 & $\sim 9$ & 1.8 \\
\hline $4 \mathrm{c}$ & PS40 & $\sim 8$ & 2.0 \\
\hline $4 \mathrm{~d}$ & PE50(red) / PS40 & $\sim 8 / \sim 7$ & 2.0 \\
\hline $5 \mathrm{a}$ & - & - & 1.0 \\
\hline
\end{tabular}




\begin{tabular}{cccc}
\hline $5 \mathrm{~b}$ & PS40 & $\sim 8$ & 1.8 \\
\hline $5 \mathrm{c}$ & PS10; PE50; PE100; PS40 & $\sim 25 ; \sim 8 ; \sim 12 ; 3$ & $0.6-5$ \\
\hline $6 \mathrm{a}-\mathrm{d}$ & - & - & $0.3-1.7$ \\
\hline $7 \mathrm{a}-\mathrm{d}$ & PE50(red) / PS40; PS40 / PS10; & & $1.5-2.0$ \\
\hline $9 \mathrm{a}$ & $\mathrm{PE} 50($ cyan) / PS40 & $\sim 6 / \sim 5$ & 2.6 \\
\hline $9 \mathrm{~b}$ & $\mathrm{PS} 140 /$ PS40 & $\sim 16 / \sim 5$ & 2.5 \\
\hline $9 \mathrm{c}$ & PE50(red) / PE50(cyan) & $\sim 7 / \sim 7$ & 2.4 \\
\hline
\end{tabular}

Movie S1. The process of formation of a particle-covered droplet using a DC electric field. Silicone oil droplet containing PE particles is formed in castor oil using a micropipette. After the application of the DC electric field, the particles are guided towards the droplet interface by electrostatic force. On the droplet's surface they are carried towards the electric equator by the electric-field-induced liquid flows. It takes several minutes for the particles to form a packed particle monolayer that covers the entire droplet surface.

Movie S2. Fabrication of a Pickering droplet with a heterogeneous shell. Two droplets (similar size) partially coated with different particles (red PE and PS) coalesce forming a Janus shell with densely packed particles.

Movie S3. Testing the mechanical properties of particle shells by inducing electric stress. When a droplet with a Janus shell is subjected to a DC electric field for $0.5 \mathrm{~s}$, the part of the shell made of PS particles deforms, whereas the thermally fused PE particle shell remains rigid.

Movie S4. The behaviour of various droplets subjected to $20 \mathrm{kHz}$ ultrasound. Pickering droplet in castor oil deforms reversibly by successive pulses and eventually falls apart. Droplet with PS particle capsule in castor oil initially remains rigid, but finally it is destroyed after being pulled into area near the ultrasonic horn. Droplet with PS particle capsule embedded in gel medium is gradually damaged by few pulses starting from the proximal side. A Pickering droplet with a patchy shell is also fragmented, although the hemispherical part composed of PE particle keeps certain integrity for longer time. In all cases the liquid payload of the capsules is spread omnidirectionally.

Movie S5. The behaviour of various droplets subjected to $1 \mathrm{MHz}$ ultrasound. In acoustic field the Pickering droplet elongates and returns to its spherical shape after the ultrasound is turned off. The liquid payload release from the droplets with PS particle capsules immersed either in castor oil or viscoelastic gel is directional and takes place through the localized shell perforation. Under the influence of ultrasound waves PS particle capsule embedded in gel does not show any translational movement as opposed to that immersed in castor oil. For a droplet with a patchy capsule application of ultrasound results in puncturing of only the PS particle shell region.

Movie S6. A pure silicone oil droplet subjected to ultrasound. The magnitude of the droplet deformation increases when the acoustic intensity is increased from 0.5 to $1.5 \mathrm{~W} / \mathrm{cm}^{2}$. At the ultrasound intensities smaller than $2 \mathrm{~W} / \mathrm{cm}^{2}$ a steady state is observed. At an ultrasound intensity of $2 \mathrm{~W} / \mathrm{cm}^{2}$, the droplet is in a non-equilibrium state, it stretches spanning almost two ultrasound wavelengths and eventually breaks apart.

Movie S7. Alignment of a capsule subjected to an applied $E$-field. The capsule consists of red PE particles and of silver conductive particles that form short chains. In the presence of an external periodic $E$-field ( $100 \mathrm{~Hz}$, in horizontal direction), the capsule undergoes electro-orientation. The size of the capsule is about $1.5 \mathrm{~mm}$. The movie was sped up 3 times. 